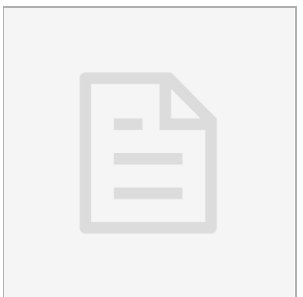

MAR 31, 2020

\section{open $\boldsymbol{\text { ACCESS }}$}

Dol:

dx.doi.org/10.17504/protocol s.io.bed3ja8n

Protocol Citation: Nathan Heath Patterson, Elizabeth Neumann, Jamie Allen, Danielle Gutierrez, Jeff Spraggins 2020. Data Processing and Preparation of MALDI IMS data.

protocols.io

https://dx.doi.org/10.17504/p

rotocols.io.bed3ja8n

License: This is an open access protocol distributed under the terms of

the Creative Commons

Attribution License, which

permits unrestricted use,

distribution, and reproduction

in any medium, provided the

original author and source

are credited

Protocol status: Working

We use this protocol and it's

working

Created: Mar 31, 2020

Last Modified: Apr 08, 2023

\section{PROTOCOL integer ID:}

34971

Keywords: HuBMAP

Keywords: HuBMAP,

Processing, MALDI IMS

\title{
(3) Data Processing and Preparation of MALDI IMS data
}

Nathan Heath Patterson ${ }^{1}$, Elizabeth Neumann ${ }^{1}, \quad$ Jamie Allen ${ }^{1}, \quad$ Danielle Gutierrez ${ }^{1}, \quad$ Jeff Spraggins $^{1}$

${ }^{1}$ Vanderbilt University

VU Biomolecular Multimodal Imaging Center / Spraggins Research Group

Human BioMolecular Atlas Program (HuBMAP) Method Developm

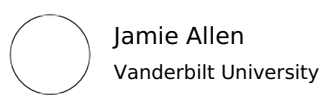

ABSTRACT

Scope:

How to process a mass spectrometry imaging data into a final imzML file.

1 For each spectrum in the data set, apply total ion current normalization by dividing the intensity values in the spectrum by the sum of the spectrum's intensity values.

4 Extract peak intensity data for every pixel using the provided identified mass list generated here (Lipid Annotation of MALDI IMS Datasets) by inverting the calibration coefficients to get the mass values in the original calibration and pulling intensity values from the Bruker .sqlite file that match these mass values.

5 Process the extracted peak list into a data table of columns: $x, y, m / z 1, m / z 2, m / z 3 \ldots$ where 1,2 , and 3 are placeholders for the identified lipid species. 
Read this table into R and create a Cardina/ MSImagingExperiment object from the pixel coordinates and peak intensity values. https://cardinalmsi.org

7 Use Cardinal's writelmzML() function to write the MSImagingExperiment object to an imzML file. 